\title{
Type Synthesis of Fully Decoupled Three Translational Parallel Mechanism with Closed-loop Units and High Stiffness
}

\author{
Shihua $\mathrm{Li}^{1,2}$, Sen Wang ${ }^{1,2}$, Haoran $\mathrm{Li}^{1,2}$, Yongjie Wang ${ }^{1,2}$, Shuang Chen ${ }^{1,2}$
}

\begin{abstract}
In this paper, a new synthesis method of fully decoupled three translational (3T) parallel mechanisms (PMs) with closed-loop units and high stiffness is proposed based on screw theory. Firstly, a new criterion for the full decoupled of PMs is presented that the reciprocal product of the transmission wrench screw matrix and the output twist screw matrix of PMs is a diagonal matrix, and all elements on the main diagonal are nonzero constants. The forms of the transmission wrench screws are determined by the criterion. Secondly, the forms of the actuated and unactuated screws can be obtained according to their relationships with the transmission wrench screws. The basic decoupled limbs are generated by combination of the above actuated and unactuated screws. Finally, a closed-loop units construction method is investigated to apply the decoupled mechanisms in a better way on the high stiffness occasion. The closed-loop units are constructed in the basic decoupled limbs to generate a highstiffness fully decoupled 3T PM. Kinematic and stiffness analyses show that the Jacobian matrix is a diagonal matrix, and the stiffness is obviously higher than that of the orthogonal coupling mechanisms, which verifies the correctness of the proposed synthesis method. The mechanism synthesized by this method has a good application prospect in vehicle durability test platform.
\end{abstract}

Keywords: Screw theory; Three translational parallel mechanism; Full decoupled; Closed-loop units; High stiffness

\section{Introduction}

In recent years, the lower-mobility parallel mechanism (PM) has become an important issue in the field of robotics due to its advantages such as low cost, simple development of control system and high performance. It is widely used in precision machining [1], aerospace [2], micro-surgery [3], 3D printing [4], spatial positioning [5] and other fields. However, the control system of the coupled PM is relatively complex compared with the decoupled one because of the strong coupled relationship between the input and output of the coupled PM [6], and it is difficult to analyze the kinematics

\footnotetext{
$\triangle$ Shihua Li

shli@ysu.edu.cn

1 Hebei Provincial Key Laboratory of Parallel Robot and Mechatronic Systems, Yanshan University

2 School of Mechanical Engineering, Yanshan University, Qinhuangdao066004, China
}

and dynamics as well as singular positions of the mechanism [7-8]. The theoretical analysis and prototype development will be simpler if the full decoupled of the PM is realized, that is, the input and output variables represent a one-to-one correspondence relationship. Therefore, the decoupled PM has become one of the research hotspots, and many researchers have studied them to solve the problem of difficult control of the coupled mechanisms.

Based on topological structure design theory of position and orientation characteristic (POC) set, Liu et al. [9] proposed three methods for structure coupling-reducing of the 3UPS\&UP PM based on the mechanism topology theory. Briot et al. [10] presented a novel 4-DOF decoupled PM with Schoenflies motions, called the Pantopteron-4. Zhang et al. [11] proposed a novel 3-DOF decoupled spatial PM. Jin et al. [12] proposed a 2R1T partially decoupled PM based on topological structure. Qu et al. [13] proposed a new method for type synthesis of decoupled PMs with low-mobility and redundancy constraints. Zhang et al. [14] synthesized a family of fully decoupled spherical mechanisms according to the screw theory. Jin et al. [15] revealed the relationship between kinematic decoupled and basic kinematic chains, proposed a structure synthesis principle of topological decoupled mechanisms, and synthesized partially decoupled 3-degree-offreedom (DOF) PMs. Lin et al. [16] studied a new type of mechanism SLBMs with three branches, and the translational and rotational motions are partially decoupled. Gogu [17] utilized linear transformation theory to study the type synthesis of nonsingular and completely isotropic 2T2R PMs. Yang et al. [18] proposed a 3-RPRS 6-DOF decoupled PM in which the three planar motion DOFs and the other three motion DOFs are independently controlled. On the basis of the topological structure characteristics of PMs, Glazunov [19] applied screw theory to obtain several decoupled 3T PMs. Kong et al. [20] used geometric description method of forward kinematic analysis to study the translational decoupled PMs. Liu et al. [21] designed a class of two to six DOFs PMs. Using the parallelograms to realize the kinematic decoupled. Li et al. [22] proposed a R-Cube decoupled 3T PM. Briot et al. [23] synthesized a faster isotropic 3T PM. Awtar [24] proposed a geometrically decoupled XYZ flexible PM. Carricato [25] realized the type synthesis of a fully isotropic 3T1R PM. Briot et al. [26] designed a series of decoupled PMs using a 4U parallelogram mechanism. Jin et al. [27] utilized the parallelogram mechanism and its evolved structures to synthesize decoupled 2T2R PMs, the moving platforms of which have a large rotation angle. Sun et al. [28] designed a 2DOF flexible fully decoupled PM driven by piezoelectric ceramics using 4-PP double bending bars as structural joints of prism. Gao et al. [29] synthesized decoupled 3T PMs using $G_{F}$ set theory. Zhang et al. [30] performed kinematic and singularity analysis of decoupled 2T1R PMs. Qu et al. [31] 
synthesized decoupled 2T1R PMs based on screw theory and Lie group. Jin et al. [32] utilized single open chain structural feature blended with topology structure and scale parameters to design decoupled 3-DOF PMs. Li et al. [33] constructed a decoupled 3T PM through the orthogonal configuration of revolute $(\mathrm{R})$ pairs and $4 \mathrm{R}$ composite kinematic pairs in $X, Y$ and $Z$ directions.

It can be seen that the decoupled 3T PMs have been widely used in many fields, but they are difficult to be applied in the high stiffness situations. Zhao et al. [34] introduced the closed-loop structure units into the kinematic chains of the mechanism, and took the closed-loop structure as the driving unit or the connecting unit in the limb, so that the stiffness performance of the mechanism could be improved because of the scale characteristics and closed characteristics of the closed-loop structure.

For improving the stiffness of the fully decoupled PMs, a new synthesis method of the fully decoupled 3T PMs with closed-loop units is proposed. The structural form of the closed-loop unit is geometrically closed. The unit is a closed loop formed by the basic decoupled limb, the constructed limb, the moving platform and the fixed platform. The criterion of full decoupled of the 3T PM is presented, in which the reciprocal product of the output twist screws and the transmission wrench screws is a diagonal matrix (the main diagonal elements are all nonzero constants). A synthetic process is developed with the closed-loop unit construction method, and a mass of high-stiffness fully decoupled 3T PMs with closed-loop units are synthesized. The synthesized mechanism not only has the 3T fully decoupled characteristic, but also has the high stiffness, and has a good application prospect in the field of PM with high stiffness.

\section{Full decoupled condition of three translation- al parallel mechanism}

According to the definition of screw, the composition form of the screw is $\left(S ; \quad S_{0}\right)$, where $S$ is called the original part and represents the direction vector, is called the dual part and can be expressed as

$$
\boldsymbol{S}_{0}=\boldsymbol{r} \times \boldsymbol{S}+h \boldsymbol{S}
$$

where $r$ is the position vector from the origin of the coordinate to any point on $\$$, and $h$ is the pitch of the screw. The screw can also be expressed in Plücker coordinate ( $\left.\begin{array}{lllllll}L & M & N & ; & P & Q & R\end{array}\right)$. The screw has two special forms: when the pitch $h=0$, the screw degenerates into a line vector $\boldsymbol{\$}=[\boldsymbol{S} ; \boldsymbol{r} \times \boldsymbol{S}]^{\mathrm{T}}$, which can represent a motion screw of a revolute pair or a pure line force vector applied to a rigid body; when $h$ tends to infinity, the screw is changed to a couple vector $\$=[0 ; \quad S]^{\mathrm{T}}$, which can represent a motion screw of a prismatic pair or a pure couple force vector applied to a rigid body. If the two screws $\$_{1}\left(S ; S_{0}\right)$ and $\$_{2}\left(S^{r} ; S_{0}^{r}\right)$ meet the conditions

$$
\$_{1} \circ \$_{2}=\boldsymbol{S} \cdot \boldsymbol{S}_{0}^{r}+\boldsymbol{S}^{r} \cdot \boldsymbol{S}_{0}
$$

Then screw $\phi_{1}$ and screw $\phi_{2}$ are mutually anti-screws, where "o" represents the reciprocal product of the two screws, and "." represents the dot multiplication of the two vectors.

Transmission wrench screw refers to the constraint wrench screw that has zero reciprocal product with all other twist screw except the actuated screw of the limb [35]. The transmission wrench screw transmits the motion/force of the input joint to the moving platform to actuate the platform in an expected motion.

Therefore, to achieve full decoupled of the three translational directions of the PM, the translational motion in one output DOF direction is only controlled by transmission wrench screw of one limb, and it will not be affected by the other two limbs. According to the screw theory, the physical meaning of the reciprocal product of the force screw and the twist screw is the instantaneous power generated by the force screw to the twist screw. In this paper, a reciprocal product algorithm of screw matrices is defined

$$
\begin{aligned}
& A=\left[\begin{array}{c}
\$_{T 1} \\
\$_{T 2} \\
\vdots \\
\$_{T n}
\end{array}\right]_{n \times 1} \quad B=\left[\begin{array}{c}
\$_{1} \\
\$_{2} \\
\vdots \\
\$_{n}
\end{array}\right]_{1 \times n}^{\mathrm{T}} \\
& A \circ B=C=\left[\begin{array}{cccc}
\$_{T 1} \circ \$_{1} & \$_{T 1} \circ \$_{2} & \cdots & \$_{T 1} \circ \$_{n} \\
\$_{T 2} \circ \$_{1} & \$_{T 2} \circ \$_{2} & \cdots & \$_{T 2} \circ \$_{n} \\
\vdots & \vdots & \ddots & \vdots \\
\$_{T n} \circ \$_{1} & \$_{T n} \circ \$_{2} & \cdots & \$_{T n} \circ \$_{n}
\end{array}\right]_{n \times n}
\end{aligned}
$$

where $A$ is a matrix of transmission wrench screws, $B$ is a matrix of output twist screws, and $\mathrm{C}$ is the reciprocal product matrix of $A$ and $B$. The elements in row $i$ and column $j$ in matrix $C$ can be expressed as

$$
(A \circ B)_{i j}=\$_{T i} \circ \$_{j}
$$

where $\$_{T i}$ is the transmission wrench screw of the $i$ limb, and $\$_{j}$ is the output twist screw of the $j$ limb.

For a fully decoupled PM, there is only instantaneous power between the transmission wrench screw and the corresponding output twist screw. Based on this, the condition for full decoupled of the 3T PM is proposed: the instantaneous power matrix is obtained by reciprocal product of the transmission wrench screw matrix of the mechanism and the corresponding output twist screw matrix, and it is a diagonal matrix (none of the main diagonal elements is zero), as shown in Eq. (6)

$$
\left[\begin{array}{l}
\boldsymbol{\$}_{T 1} \\
\boldsymbol{\$}_{T 2} \\
\boldsymbol{\$}_{T 3}
\end{array}\right] \circ\left[\begin{array}{l}
\boldsymbol{\$}_{1} \\
\boldsymbol{\$}_{2} \\
\boldsymbol{\$}_{3}
\end{array}\right]^{\mathrm{T}}=\left[\begin{array}{ccc}
\boldsymbol{\$}_{T 1} \circ \boldsymbol{\$}_{1} & 0 & 0 \\
0 & \boldsymbol{\$}_{T 2} \circ \boldsymbol{\$}_{2} & 0 \\
0 & 0 & \boldsymbol{\$}_{T 3} \circ \boldsymbol{\$}_{3}
\end{array}\right]_{3 \times 3}
$$

\section{Construction method of closed-loop units}

In this paper, a closed-loop unit is constructed in the limb to increase the stiffness of the fully decoupled PMs, and it will not change the decoupled characteristic. According to the relationship between the output twist screw and transmission wrench screw in the fully decoupled condition, the transmission wrench screw of the limb is determined. Next, the basic decoupled limb is synthesized by combining the construction method of the actuated screw and the unactuated screw. Finally, the limb which is completely consistent and driven synchronously with the basic decoupled limb is generated, and it forms a closed-loop unit with the basic decoupled limb. The construction method of the closed-loop unit needs to consider the types of kinematic pairs which are directly connected to the moving platform and the fixed 
platform. There are two types of kinematic pairs, prismatic pair and revolute pair. The spatial geometric relationship of two kinematic pairs of the basic decoupled limb and the constructed limb are also considered. The spatial geometric relationship of kinematic pairs can be divided into three cases: coaxial, parallel and share. When the driving pairs are shared, the redundant actuation is not necessary to be added. When the driving pairs are coaxial or parallel, redundant actuation synchronized with the actuation of the basic decoupled limb needs to be added in the construction limb.

The construction method of the closed-loop units is as follows.

(1) Using prismatic pair as the driving pair

When the kinematic pair directly connected to the moving platform in the basic decoupled limb is $\mathrm{R}$ pair, the $\mathrm{R}$ pair connected to the moving platform in the constructed limb can be coaxial or parallel to it. When the $\mathrm{R}$ pairs are coaxial, the driving pair in the constructed limb and the one in the basic decoupled limb can be coaxial (As shown in Figure 1(a)), or shared (As shown in Figure 1(b)). There is no need to add redundant actuation only when the driving pair is shared. When the driving pair in the constructed limb is coaxial to the one in the basic decoupled limb, the redundant actuation should be added into the closed-loop unit, and it is synchronized with the actuation of the basic decoupled limb. When the $\mathrm{R}$ pairs are parallel, the driving pair in the constructed limb is parallel to the one in the basic decoupled limb. The redundant actuation should be added into the closed-loop unit, and it is synchronized with the actuation of the basic decoupled limb, as shown in Figure 1(c).

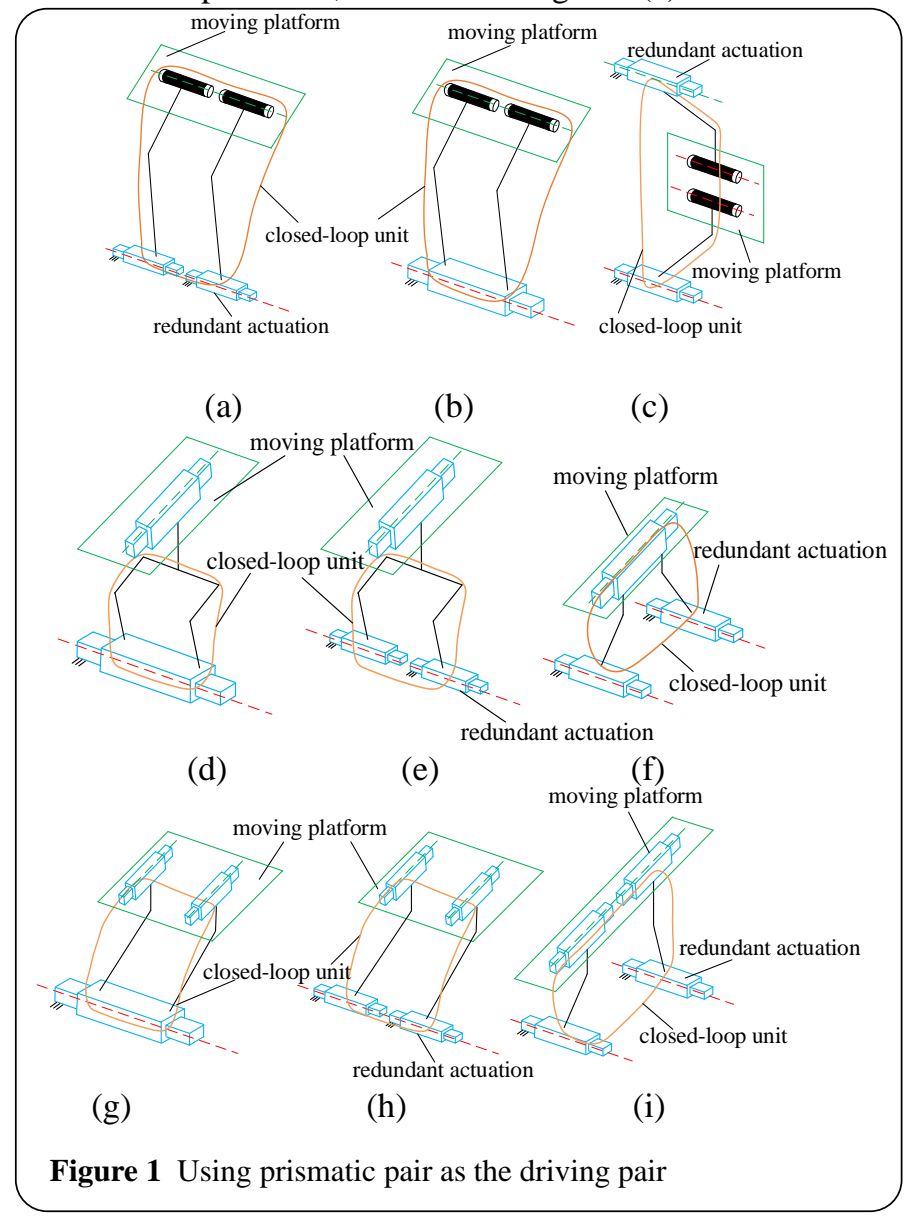

When the kinematic pair directly connected to the moving platform in the basic decoupled limb is prismatic $(\mathrm{P})$ pair, the $\mathrm{P}$ pair connected to the moving platform in the constructed limb is coaxial or parallel to it. They can also share the same $\mathrm{P}$ pair.

When the $\mathrm{P}$ pair is shared, the driving pair in the constructed limb and the one in the basic decoupled limb can be shared (As shown in Figure 1(d)), coaxial (As shown in Figure 1(e)) or parallel (As shown in Figure 1(f)). There is no need to add redundant actuation only when the driving pair is shared.

When the $\mathrm{P}$ pairs are parallel, the driving pair in the constructed limb and the one in the basic decoupled limb can be shared (As shown in Figure 1(g)), or coaxial (As shown in Figure 1(h)). When the driving pair in the constructed limb is coaxial to the one in the basic decoupled limb, the redundant actuation should be added into the closed-loop unit, and it is synchronized with the actuation of the basic decoupled limb.

When the $\mathrm{P}$ pairs are coaxial, the driving pair in the constructed limb is parallel to the one in the basic decoupled limb. In the above case, the redundant actuation should be added into the closed-loop unit, and it is synchronized with the actuation of the basic decoupled limb, as shown in Figure 1(i).

(2) Using revolute pair as the driving pair

When the kinematic pair directly connected to the moving platform in the basic decoupled limb is $\mathrm{R}$ pair, the $\mathrm{R}$ pair connected to the moving platform in the constructed limb can only be parallel to it. When two $\mathrm{R}$ pairs are parallel, the driving pair in the constructed limb and the one in the basic decoupled limb can be coaxial (As shown in Figure 2(a)), parallel (As shown in Figure 2(b)) or shared (As shown in Figure 2(c)). There is no need to add redundant actuation only when the driving pair is shared.

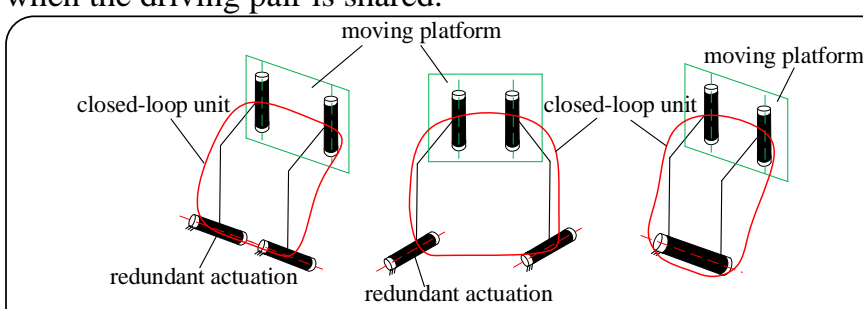

(a)

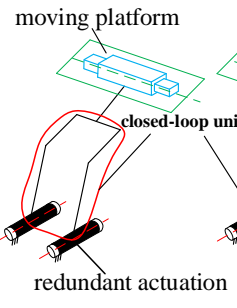

(d) (b) moving platform moving platform (c)

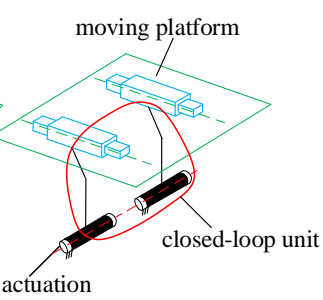

(e) (f)
Figure 2 Using revolute pair as the driving pair

When the kinematic pair directly connected to the moving platform in the basic decoupled limb is $\mathrm{P}$ pair, the $\mathrm{P}$ pair connected to the moving platform in the constructed limb is coaxial (As shown in Figure 2(e)), parallel (As shown in Figure 2(f)) or shared with it (As shown in Figure 2(d)). When the $\mathrm{P}$ pair is shared or coaxial, the driving pair in the constructed limb is parallel to the one in the basic decoupled limb. When the $\mathrm{P}$ pairs are parallel, the driving pair in the constructed limb is coaxial to the one in the basic decoupled limb. In the above case, the redundant actuation should be 
added into the closed-loop unit, and it is synchronized with the actuation of the basic decoupled limb.

\section{A new synthetic method of three translational fully decoupled parallel mechanism with closed- loop units}

From the above analysis, the synthesis process of the fully decoupled 3T PMs is developed as follows, and the flow chart is shown in Figure 3.

(1) Based on the DOF and decoupled requirements of the 3T PM, a full decoupled condition is proposed that the reciprocal product of the transmission wrench screw matrix and the output twist screw matrix of the limb is a diagonal matrix (none of the main diagonal elements are zero), and the forms of transmission wrench screw of each limb are determined.

(2) The form of the actuated screw in the limb is obtained according to the criterion that the reciprocal product of the transmission wrench screw and the actuated screw in the same limb is a nonzero constant.

(3) The form of the unactuated screw is determined according to the criterion that the reciprocal product of the transmission wrench screw and all the other kinematic screws except the actuated screw in the same limb is equal to zero.

(4) On the basis of the type of actuated screw and unactuated screw, the connectivity of the limb and the different order of the kinematic pairs, the basic decoupled limb is synthesized.

(5) The limb which is completely consistent and driven synchronously with the basic decoupled limb is constructed by the closed-loop unit construction method. Then, a closed-loop unit with a basic decoupled limb is constituted to improve the stiffness performance of the mechanism, and the type synthesis of the fully decoupled 3T PM with high stiffness is realized.

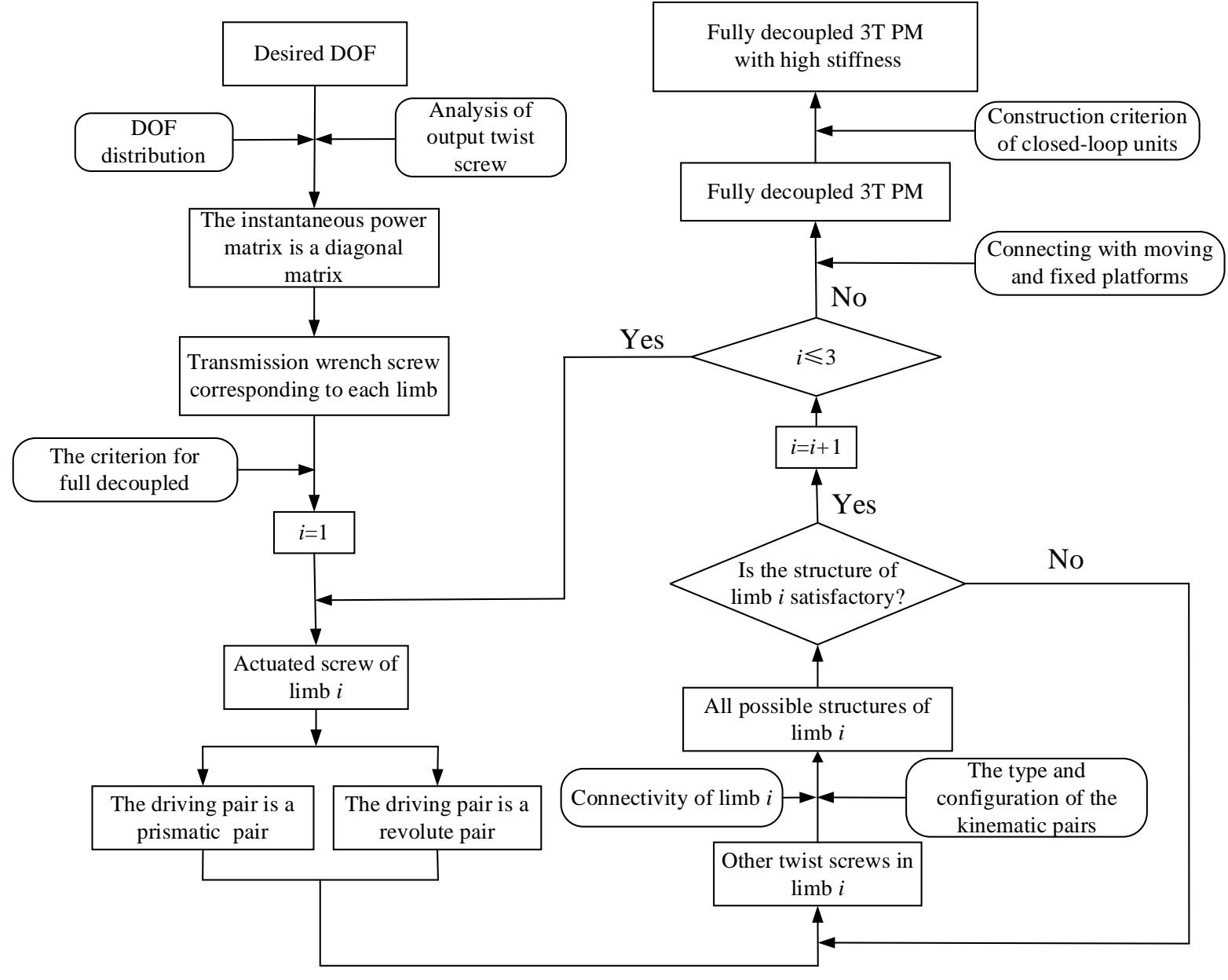

Figure 3 Synthesis flow chart of three translational fully decoupled parallel mechanism

\section{Synthesis of three translational fully decoupl- ed parallel mechanism with closed-loop units}

Decoupled limbs need to be constructed firstly according to the expected DOF and decoupled characteristic for synthesis of the high-stiffness decoupled 3T PMs with the closed-loop units. Under the condition that the decoupled characteristic of the mechanism is not changed, a closed-loop unit is formed with the basic decoupled limb and the constructed limb based on the closed-loop unit construction method. Ultimately, a high-stiffness decoupled 3T PM with the closed-loop units is constituted.

\subsection{Configuration synthesis of limb 1}

As shown in Figure 4,o-xyz is the base coordinate system, $\$_{1}, \$_{2}, \$_{3}$ are the output twist screws of the three motion 
directions of the moving platform. $\$_{T i}$ is the transmission wrench screw of limb $i$ acting on the moving platform. According to the criterion for the full decoupled of PMs, each limb of the fully decoupled 3T PM only controls one output translation motion. Assuming that the three limbs control the output motion in the three directions of $x, y$ and $z$ axes respectively, and the output twist screw is a translational screw with infinite pitch in the same direction as the output motion. Therefore, $\$_{1}, \$_{2}, \$_{3}$ are expressed as

$$
\begin{aligned}
& \$_{1}=\left(\begin{array}{lllll}
0 & 0 & 0 ; 1 & 0 & 0
\end{array}\right) \\
& \$_{2}=\left(\begin{array}{lllll}
0 & 0 & 0 ; 0 & 1 & 0
\end{array}\right) \\
& \$_{3}=\left(\begin{array}{lllll}
0 & 0 & 0 ; 0 & 0 & 1
\end{array}\right)
\end{aligned}
$$

Based on the decoupled criterion, the output translational motion along the $x$-axis direction is only provided by limb 1 , that is, the reciprocal product of the transmission wrench screw of the limb and the corresponding output twist screw is not zero, and the reciprocal product with the output twist screw controlled by other limbs is equal to zero.

Provided that the transmission wrench screw of limb 1 is

$$
\$_{T 1}=\left(\begin{array}{lllll}
a_{1} & b_{1} & c_{1} ; d_{1} & e_{1} & f_{1}
\end{array}\right)
$$

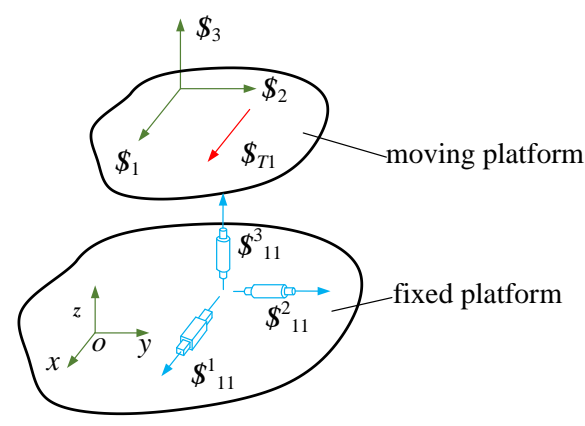

Figure 4 Schematic diagram of transmission screw and actuated screw of limb 1

If $\boldsymbol{\$}_{T 1} \circ \$_{1} \neq 0, \$_{T 1} \circ \$_{2}=0, \$_{T 1} \circ \$_{3}=0$ is satisfied, the following equations can be deduced

$$
\begin{aligned}
& a_{1}+d_{1} \cdot 0+e_{1} \cdot 0+f_{1} \cdot 0 \neq 0 \\
& b_{1}+d_{1} \cdot 0+e_{1} \cdot 0+f_{1} \cdot 0=0 \\
& c_{1}+d_{1} \cdot 0+e_{1} \cdot 0+f_{1} \cdot 0=0
\end{aligned}
$$

It can be solved from Eq. (9) that $b_{1}, c_{1}$ must be zero and $a_{1} \neq 0$. The Eq.(9) will hold true no matter what the values of $\phi_{T i}$ are. The value of $d_{1}=0, e_{1} \neq 0, f_{1} \neq 0$ is taken. The transmission wrench screw does not pass through the origin of the coordinate. In this case, the transmission wrench screw acting on the moving platform of limb 1 can be finally determined as

$$
\$_{T 1}=\left(\begin{array}{lllll}
1 & 0 & 0 ; 0 & e_{1} & f_{1}
\end{array}\right)
$$

It can be known from above analysis that the relationship between transmission wrench screw of each limb and output twist screw of the moving platform of the fully decoupled 3T PM is that the transmission wrench screw of each limb is coplanar with the output twist screw of the corresponding limb, and perpendicular to the output twist screw of other limbs.

The transmission wrench screw $\$_{T 1}$ of limb 1 is the force screw in the direction of the $x$-axis, which is in the same direction as the output motion controlled by the limb.
Similarly, limb 2 controls the output motion in the $y$-axis direction, and limb 3 controls the output motion in the $z$-axis direction. Thus, the transmission wrench screw of limb 2 is the force screw in the $y$-axis direction, and the transmission wrench screw of limb 3 is the force screw in the $z$-axis direction. The transmission wrench screw of the limb acting on the moving platform can only be the linear force vector in the same direction as the output twist screw of the limb.

According to the condition that the reciprocal product of the transmission wrench screw $\$_{T i}$ and the actuated screw $\$_{i 1}$ in the same kinematic limb is a nonzero constant: $\$_{T i} \circ \$_{i 1} \neq 0(i$ is the $i \operatorname{limb}$ ), the actuated screw of each limb is constructed. Take limb 1 as an example.

Provided that the actuated screw $\$_{11}$ of limb 1 is

$$
\$_{11}=\left(\begin{array}{lllll}
l_{1} & m_{1} & n_{1} ; u_{1} & v_{1} & w_{1}
\end{array}\right)
$$

If $\$_{T 1} \circ \$_{11} \neq 0$, the following equation needs to be satisfied

$$
l_{1} \cdot 0+m_{1} \mathrm{e}_{1}+n_{1} f_{1}+u_{1}+v_{1} \cdot 0+w_{1} \cdot 0 \neq 0
$$

The Eq.(12) will hold true no matter what the values of $l_{1}, v_{1}, w_{1}$ are.

If the pitch of $\$_{11}$ is infinitely large, there's only one case, which is $m_{1}=0, n_{1}=0, u_{1} \neq 0$.

$$
\$_{11}^{1}=\left(\begin{array}{lllll}
0 & 0 & 0 ; 1 & 0 & 0
\end{array}\right)
$$

At this moment, the driving pair is a $\mathrm{P}$ pair with the axis along the $x$-axis direction.

If the pitch of $\$_{11}$ is zero, there are two cases

A. $m_{1} \neq 0, n_{1}=0, u_{1} \neq 0$

$$
\$_{11}^{2}=\left(\begin{array}{ccccc}
0 & 1 & 0 ; u_{1} & 0 & w_{1}
\end{array}\right)
$$

At this moment, the driving pair is a $\mathrm{R}$ pair with the axis along the $y$-axis direction.

B. $m_{1}=0, n_{1} \neq 0, u_{1} \neq 0$

$$
\$_{11}^{3}=\left(\begin{array}{lllll}
0 & 0 & 1 ; u_{1} & v_{1} & 0
\end{array}\right)
$$

At this moment, the driving pair is a $\mathrm{R}$ pair with the axis along the $z$-axis direction.

To sum up, the transmission wrench screw $\$_{T 1}$ of limb 1 acting on the moving platform and all possible actuated screws $\$_{11}^{1} 、 \$_{11}^{2} 、 \$_{11}^{3}$ are shown in Figure 4 .

According to the condition that the reciprocal product of the transmission wrench screw $\$_{T i}$ and the unactuated kinematic screw except the actuated screw in the same limb is equal to zero: $\$_{T i} \circ \$_{i j}=0$ ( $i$ represents the $i$ limb, $j$ represents other kinematic screws in the limb except the actuated screw and $1<j \leq 5)$, the unactuated screw of limb 1 is

$$
\$_{1 j}=\left(\begin{array}{lllll}
l_{1 j} & m_{1 j} & n_{1 j} ; u_{1 j} & v_{1 j} & w_{1 j}
\end{array}\right)
$$

If $\$_{T 1} \circ \$_{1 j}=0$, the following equation can be deduced

$$
l_{1 j} \cdot 0+m_{1 j} e_{1}+n_{1 j} f_{1}+u_{1 j}+v_{1 j} \cdot 0+w_{1 j} \cdot 0=0
$$

There are two cases for Eq. (17) to be true. In the first case, the conditions $m_{1 j}=0, n_{1 j}=0, u_{1 j}=0$ need to be satisfied, the unactuated screw in the limb may be a line vector in the $x$-axis direction or a couple vector in the direction of $y$ and $z$ axis. In the second case, $\$_{1 j}$ is a line vector which always intersects with $\$_{T 1}$, and both cases can coexist in the same limb.

Since the limbs should contain all the motion 
characteristics of the moving platform to achieve the expected output motion of the moving platform, each limb must have translational DOF in at least $x, y$ and $z$ directions, and the connectivity of the limb should be at least 3 . When the actuation is a $\mathrm{R}$ pair, $2 \mathrm{R}$ parallel sub-chain can be used as the driving pair, But the original limb should contain translational DOF whose direction is perpendicular to the axis of the $\mathrm{R}$ pair (A $2 \mathrm{R}$ parallel sub-chain can be considered as a linear combination of a $\mathrm{R}$ pair and a $\mathrm{P}$ pair). Moreover, there should be one translational DOF parallel to the negative motion direction of the $2 \mathrm{R}$ sub-chain. Negative motion direction is the non-corresponding output DOF direction of the two translational DOFs vertical to the axis of $\mathrm{R}$ pair. As shown in Table 1, the types of unactuated screw of limb 1 and their satisfying conditions are discussed according to the types of driving pairs of limb 1.

In this paper, only the connectivity of 3,4 and 5 of limb 1 are considered. According to the types of actuated and unactuated screws, the configuration orientation and connectivity of limb 1, all possible kinematic limb structures are synthesized, as shown in Table 2 . In Table 2, $\mathrm{P}$ represents the prismatic pair, $\mathrm{R}$ represents the revolute pair, $x, y, z$ represent the axis directions of the prismatic pair or revolute pair, $\mathrm{C}$ represents the cylindrical pair, $\mathrm{U}$ represents the universal pair, and the lower angle symbol $n$ indicates that the direction of the prismatic pair is orthogonal to the directions of the adjacent two $R$ pairs. The $2 R$ parallel sub-chain can be replaced by a parallelogram structure $\mathrm{Pa}$, which is composed of four $\mathrm{R}$ pairs in the same direction as the actuated screw to improve bearing capacity of the limb. To simplify the structure, suppose that the axes of the adjacent kinematic pairs in the limb are parallel or perpendicular to each other. This configuration can be designed and reformed according to the specific application conditions in the actual engineering. Schematic diagrams of typical limb configurations and structures are shown in Table 3. The basic decoupled limbs in Table 2 and Table 3 are not the final configurations. The 3T fully decoupled PMs with closed-loop units are formed by the redundant limbs constructed by the new method of closedloop unit construction and the basic decoupled limbs.

\section{Table 1 Unactuated screw types in limb 1 and their satisfying conditions}

\section{Type of driving pair}

(1) Line vector screws in the same direction as the actuated screw $\$_{11}^{1}$. The number of such screws is at least 2 . The maximal linear independent number of spatial parallel line vectors is 3 , and the maximum number of such screws is 3 .

When the pitch of actuated screw is infinitely large, the driving pair is a prismatic pair in the direction of the $x$-axis

(2) Couple vector screws perpendicular to the actuated screw $\$_{11}^{1}$. Such screws cannot be parallel to each other in space. The maximum linearly independent number of plane intersection couple vectors is 2 , and the number of such screws is no more than 2 .
(3) Line vector screws intersecting with the transmission wrench screw $\$_{T 1}$. The number of such screws is at most 2 . When the number is 2 , the screw must intersect with $\boldsymbol{\$}_{T 1}$ at a point. Such screws have no effect on the output motion of the mechanism, so they are also called inert pairs.

(1) The number of line vector screws parallel to the $x$-axis is at least 2 and at most 3 . No other line vector screws with different directions can be added between the screws.

(1) When the number of the screw is 1 , if the two ends of the screw are in the same direction as the line vectors connected to it, the axis of the couple vector should be perpendicular to and coplanar with the two end line vectors. If the two ends of the line vector connected with it are orthogonal, the axis direction of the couple vector can only be along the $y$-axis or $z$ axis direction, and the couple vector can also be directly connected with the moving platform.

(2) When the number of the screw is 2 , the two couple vectors are along the $y$ direction and the $z$ direction, and at most one couple vector in the same direction as the driving pair is arranged in the $2 \mathrm{R}$ parallel subchain. When a couple vector is set in the $2 \mathrm{R}$ parallel sub-chain, another couple vector is arranged between the $2 \mathrm{R}$ parallel sub-chain and the moving platform. Otherwise, two couple vectors are arranged between the $2 \mathrm{R}$ parallel sub-chain and the moving platform.

(3) Line vector screws intersecting with the transmission wrench screw $\boldsymbol{\$}_{T 1}$ and perpendicular to the actuated screw. The number of such screws is at most 2, and they are inert screws.

\subsection{Configuration synthesis of limb 2}

If limb 2 provides $y$-direction output motion, provided that the transmission wrench screw of limb 2 is

$$
\$_{T 2}=\left(\begin{array}{lllll}
a_{2} & b_{2} & c_{2} ; d_{2} & e_{2} & f_{2}
\end{array}\right)
$$

If condition $\$_{T 2} \circ \$_{2} \neq 0, \$_{T 2} \circ \$_{1}=0, \$_{T 2} \circ \$_{3}=0$ is satisfied, the following equations can be deduced 


$$
\begin{aligned}
& b_{2}+d_{2} \cdot 0+e_{2} \cdot 0+f_{2} \cdot 0 \neq 0 \\
& a_{2}+d_{2} \cdot 0+e_{2} \cdot 0+f_{2} \cdot 0=0 \\
& c_{2}+d_{2} \cdot 0+e_{2} \cdot 0+f_{2} \cdot 0=0
\end{aligned}
$$

It can be solved from Eq. (19) that $a_{2}, c_{2}$ must be zero and $b_{2} \neq 0$. The Eq.(19) will hold true no matter what the values of $d_{2}, e_{2}, f_{2}$ are. The value of $e_{2}=0, d_{2} \neq 0, f_{2} \neq 0$ is taken. In this case, the transmission wrench screw acting on the moving platform of limb 2 can be finally determined as

$$
\$_{T 2}=\left(\begin{array}{lllll}
0 & 1 & 0 ; d_{2} & 0 & f_{2}
\end{array}\right)
$$

The transmission wrench screw $\$_{T 2}$ of limb 2 is the force screw in the $y$-axis direction, which is in the same direction as the output motion of the limb. According to the conditions that the reciprocal product of the transmission wrench screw $\$_{T i}$ and the actuated screw $\boldsymbol{\$}_{i 1}$ in the same kinematic limb is a nonzero constant: $\boldsymbol{\$}_{T i} \circ \$_{i 1} \neq 0$ ( $i$ is the $i \operatorname{limb}$ ), the actuated screw of each limb is obtained.

Provided that the actuated screw $\$_{21}$ of limb 2 is

$$
\$_{21}=\left(\begin{array}{lllll}
l_{2} & m_{2} & n_{2} ; u_{2} & v_{2} & w_{2}
\end{array}\right)
$$

If $\$_{T 2} \circ \$_{21} \neq 0$, the following equation needs to be satisfied

$$
l_{2} \cdot d_{2}+m_{2} \cdot 0+n_{2} f_{2}+u_{2} \cdot 0+v_{2}+w_{2} \cdot 0 \neq 0
$$

The Eq.(22) will hold true no matter what the values of $m_{2}, u_{2}, w_{2}$ are.

If the pitch of $\$_{21}$ is infinitely large, there's only one case, $l_{2}=0, n_{2}=0, v_{2} \neq 0$

$$
\$_{21}^{1}=\left(\begin{array}{lllll}
0 & 0 & 0 ; 0 & 1 & 0
\end{array}\right)
$$

At this moment, the driving pair is a $\mathrm{P}$ pair with the axis along the $y$-axis direction.

If the pitch of $\$_{21}$ is zero, there are two cases, which are

$$
\begin{aligned}
\text { A. } l_{2} \neq 0, n_{2}=0, v_{2} & \neq 0 \\
& \$_{21}^{2}=\left(\begin{array}{lllll}
1 & 0 & 0 ; 0 & v_{2} & w_{2}
\end{array}\right)
\end{aligned}
$$

\begin{tabular}{|c|c|c|c|c|c|}
\hline $\begin{array}{c}\text { Type of } \\
\text { driving pair }\end{array}$ & $\begin{array}{l}\text { Connectivity } \\
\text { of the limb }\end{array}$ & Limb configuration & $\begin{array}{c}\text { Basic decoupled } \\
\text { limb configuration }\end{array}$ & $\begin{array}{l}\text { Compound kinematic } \\
\text { pair }\end{array}$ & $\begin{array}{c}\text { Pa limb } \\
\text { structure }\end{array}$ \\
\hline \multirow{11}{*}{$\begin{array}{c}\text { Driving pair } \\
\text { is a } \\
\text { prismatic } \\
\text { pair }\end{array}$} & 3 & $3 \mathrm{P}$ & $\mathrm{P}_{x} \mathrm{P}_{y} \mathrm{P}_{z}$ & & \\
\hline & \multirow{8}{*}{4} & \multirow{3}{*}{ 3P1R } & $\mathrm{P}_{x} \mathrm{P}_{y} \mathrm{P}_{z} \mathrm{R}_{y}$ & & \\
\hline & & & $\mathrm{P}_{x} \mathrm{P}_{y} \mathrm{P}_{z} \mathrm{R}_{z}$ & & \\
\hline & & & $\mathrm{P}_{x} \mathrm{R}_{x} \mathrm{P}_{n} \mathrm{R}_{x}$ & $\mathrm{C}_{x} \mathrm{P}_{n} \mathrm{R}_{x}$ & \\
\hline & & \multirow{4}{*}{$2 \mathrm{P} 2 \mathrm{R}$} & $\mathrm{P}_{x} \mathrm{R}_{x} \mathrm{R}_{x} \mathrm{P}_{y}$ & $\mathrm{C}_{x} \mathrm{R}_{x} \mathrm{P}_{y}$ & \\
\hline & & & $\mathrm{P}_{x} \mathrm{P}_{y} \mathrm{R}_{x} \mathrm{R}_{x}$ & & \\
\hline & & & $\mathrm{P}_{x} \mathrm{R}_{x} \mathrm{R}_{x} \mathrm{P}_{z}$ & $\mathrm{C}_{x} \mathrm{R}_{x} \mathrm{P}_{z}$ & \\
\hline & & & $\mathrm{P}_{x} \mathrm{P}_{z} \mathrm{R}_{x} \mathrm{R}_{x}$ & & \\
\hline & & 1P3R & $\mathrm{P}_{x} \mathrm{R}_{x} \mathrm{R}_{x} \mathrm{R}_{x}$ & $\mathrm{C}_{x} \mathrm{R}_{x} \mathbf{R}_{x}$ & \\
\hline & 5 & 1P4P & $\mathrm{P}_{x} \mathrm{R}_{x} \mathrm{R}_{x} \mathrm{R}_{x} \mathrm{R}_{z}$ & $\mathrm{C}_{x} \mathrm{R}_{x} \mathrm{R}_{x} \mathrm{R}_{z}$ & \\
\hline & $J$ & $114 \pi$ & $\mathrm{P}_{x} \mathrm{R}_{x} \mathrm{R}_{x} \mathrm{R}_{x} \mathrm{R}_{y}$ & $\mathrm{C}_{x} \mathrm{R}_{x} \mathrm{R}_{x} \mathrm{R}_{y}$ & \\
\hline \multirow{14}{*}{$\begin{array}{c}\text { Driving pair } \\
\text { is a revolute } \\
\text { pair }\end{array}$} & \multirow{7}{*}{4} & \multirow{2}{*}{$2 \mathrm{P} 2 \mathrm{R}$} & $\mathrm{R}_{y} \mathrm{R}_{y} \mathrm{P}_{y} \mathrm{P}_{z}$ & $\mathrm{R}_{y} \mathrm{C}_{y} \mathrm{P}_{z}$ & $\mathrm{PaP}_{y} \mathrm{P}_{z}$ \\
\hline & & & $\mathrm{R}_{y} \mathrm{P}_{y} \mathrm{R}_{y} \mathrm{P}_{z}$ & $\mathrm{C}_{y} \mathrm{R}_{y} \mathrm{P}_{z} 、 \mathrm{R}_{y} \mathrm{C}_{y} \mathrm{P}_{z}$ & \\
\hline & & \multirow{4}{*}{ 2P3R } & $\mathrm{R}_{y} \mathrm{R}_{y} \mathrm{P}_{y} \mathrm{P}_{z} \mathrm{R}_{x}$ & $\mathrm{R}_{y} \mathrm{C}_{y} \mathrm{P}_{z} \mathrm{R}_{x}$ & $\mathrm{PaP}_{y} \mathrm{P}_{z} \mathrm{R}_{x}$ \\
\hline & & & $\mathrm{R}_{y} \mathrm{P}_{y} \mathrm{R}_{y} \mathrm{P}_{z} \mathrm{R}_{x}$ & $\mathrm{C}_{y} \mathrm{R}_{y} \mathrm{P}_{z} \mathrm{R}_{x} 、 \mathrm{R}_{y} \mathrm{C}_{y} \mathrm{P}_{z} \mathrm{R}_{x}$ & \\
\hline & & & $\mathrm{R}_{y} \mathrm{R}_{y} \mathrm{P}_{y} \mathrm{P}_{z} \mathrm{R}_{z}$ & $\mathrm{R}_{y} \mathrm{C}_{y} \mathrm{P}_{z} \mathrm{R}_{z}$ & $\mathrm{PaP}_{y} \mathrm{P}_{z} \mathrm{R}_{z}$ \\
\hline & & & $\mathrm{R}_{y} \mathrm{P}_{y} \mathrm{R}_{y} \mathrm{P}_{z} \mathrm{R}_{z}$ & $\mathrm{C}_{y} \mathrm{R}_{y} \mathrm{P}_{z} \mathrm{R}_{z}, \mathrm{R}_{y} \mathrm{C}_{y} \mathrm{P}_{z} \mathrm{R}_{z}$ & \\
\hline & & \multirow{5}{*}{ 1P4R } & $\mathrm{R}_{y} \mathrm{R}_{y} \mathrm{R}_{x} \mathrm{R}_{x} \mathrm{P}_{z}$ & $\mathrm{R}_{y} \mathrm{U}_{y x} \mathrm{R}_{x} \mathrm{P}_{z}$ & $\mathrm{PaR}_{x} \mathrm{R}_{x} \mathrm{P}_{z}$ \\
\hline & \multirow{7}{*}{5} & & $\mathrm{R}_{y} \mathrm{R}_{y} \mathrm{P}_{z} \mathrm{R}_{x} \mathrm{R}_{x}$ & & $\mathrm{PaP}_{z} \mathrm{R}_{x} \mathrm{R}_{x}$ \\
\hline & & & $\mathrm{R}_{y} \mathrm{R}_{y} \mathrm{R}_{x} \mathrm{P}_{n} \mathrm{R}_{x}$ & $\mathrm{R}_{y} \mathrm{U}_{y z} \mathrm{P}_{n} \mathrm{R}_{x}$ & $\mathrm{PaR}_{x} \mathrm{P}_{n} \mathrm{R}_{x}$ \\
\hline & & & $\mathrm{R}, \mathrm{P} R \mathrm{R} R$ & $\mathrm{C}_{y} \mathrm{R}_{y} \mathrm{R}_{x} \mathrm{R}_{x}$, & \\
\hline & & & 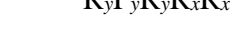 & $\mathrm{C}_{y} \mathrm{U}_{y x} \mathrm{R}_{x} 、 \mathrm{R}_{y} \mathrm{C}_{y} \mathrm{R}_{x} \mathrm{R}_{x}$ & \\
\hline & & \multirow{3}{*}{$5 \mathrm{R}$} & $\mathrm{R}_{y} \mathrm{R}_{y} \mathrm{P}_{y} \mathrm{R}_{x} \mathrm{R}_{x}$ & & $\mathrm{PaP}_{y} \mathrm{R}_{x} \mathrm{R}_{x}$ \\
\hline & & & $\mathrm{R}_{y} \mathrm{R}_{y} \mathrm{R}_{x} \mathrm{R}_{x} \mathrm{P}_{y}$ & $\mathrm{R}_{y} \mathrm{U}_{y x} \mathrm{R}_{x} \mathrm{P}_{y}$ & $\mathrm{PaR}_{x} \mathrm{R}_{x} \mathrm{P}_{y}$ \\
\hline & & & $\mathrm{R}_{y} \mathrm{R}_{y} \mathrm{R}_{x} \mathrm{R}_{x} \mathbf{R}_{x}$ & $\mathrm{R}_{y} \mathrm{U}_{y x} \mathrm{R}_{x} \mathrm{R}_{x}$ & $\mathrm{PaR}_{x} \mathrm{R}_{x} \mathrm{R}_{x}$ \\
\hline
\end{tabular}

At this moment, the driving pair is a $\mathrm{R}$ pair with the axis along the $x$-axis direction.

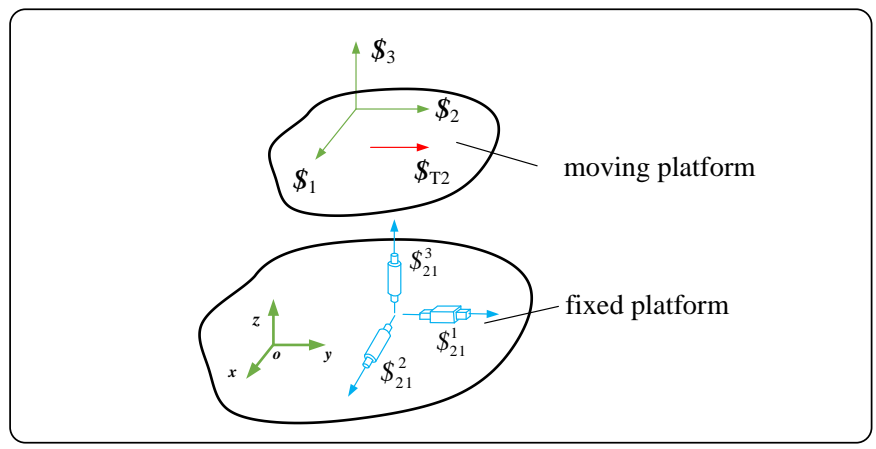

Figure 5 Schematic diagram of transmission force screw and actuated screw of limb 2

\section{Table 2 Configuration of limb 1}


Table3 Typical configuration and structure diagram of limb 1

\begin{tabular}{|c|c|c|c|c|}
\hline $\begin{array}{l}\text { Basic decoupled limb } \\
\text { configuration }\end{array}$ & $\mathrm{C}_{x} \mathrm{P}_{n} \mathrm{R}_{x}$ & $\mathrm{C}_{x} \mathrm{R}_{x} \mathrm{R}_{x}$ & $\mathrm{P}_{x} \mathrm{R}_{x} \mathrm{R}_{x} \mathrm{P}_{y}$ & $\mathrm{R}_{y} \mathrm{P}_{y} \mathrm{R}_{y} \mathrm{P}_{z}$ \\
\hline \multicolumn{5}{|l|}{ Structure diagram } \\
\hline $\begin{array}{c}\text { Basic decoupled limb } \\
\text { configuration }\end{array}$ & $\mathrm{R}_{y} \mathrm{R}_{y} \mathrm{R}_{x} \mathrm{P}_{n} \mathbf{R}_{x}$ & $\mathrm{C}_{y} \mathrm{R}_{y} \mathrm{R}_{x} \mathrm{R}_{x}$ & $\mathbf{R}_{y} \mathbf{R}_{y} \mathbf{R}_{x} \mathbf{R}_{x} \mathbf{R}_{x}$ & $\mathrm{R}_{y} \mathbf{R}_{y} \mathbf{R}_{x} \mathbf{R}_{x} \mathrm{P}_{y}$ \\
\hline
\end{tabular}

B. $l_{2}=0, n_{2} \neq 0, v_{2} \neq 0$

$$
\$_{21}^{3}=\left(\begin{array}{lllll}
0 & 0 & 1 ; u_{2} & v_{2} & 0
\end{array}\right)
$$

At this moment, the driving pair is a $\mathrm{R}$ pair with the axis along the $z$-axis direction. To sum up, the transmission wrench screw $\$_{T 2}$ of limb 2 acting on the moving platform and all possible actuated screws $\boldsymbol{\$}_{21}^{1} 、 \boldsymbol{\$}_{21}^{2} 、 \boldsymbol{\$}_{21}^{3}$ are shown in Figure 5.

On the basis of the condition that the reciprocal product of the transmission wrench screw $\$_{T i}$ and the unactuated kinematic screw except the actuated screw in the same limb is equal to zero: $\boldsymbol{\$}_{T i} \circ \$_{i j}=0$ ( $i$ represents the $i$ limb, $j$ represents other kinematic screws in the limb except the actuated screw and $1<j \leq 5$ ), the unactuated screw of limb 2 is

$$
\$_{2 j}=\left(\begin{array}{lllll}
l_{2 j} & m_{2 j} & n_{2 j} ; u_{2 j} & v_{2 j} & w_{2 j}
\end{array}\right)
$$

If $\$_{T 2} \circ \$_{2 j}=0$, the following equation can be deduced

$$
l_{2 j} d_{2}+m_{2 j} \cdot 0+n_{2 j} f_{2}+u_{2 j} \cdot 0+v_{2 j}+w_{2 j} \cdot 0=0
$$

There are two cases for Eq. (27) to be true. In the first case, the condition $l_{2 j}=0, n_{2 j}=0, v_{2 j}=0$ needs to be satisfied. The unactuated screw can be a line vector in the $y$ axis direction or a couple vector in the direction of $x$ and $z$ axis. In the second case, $\$_{2 j}$ is a line vector which always intersects with $\$_{T 2}$, and both cases can coexist in the same limb.

The types and conditions of the unactuated kinematic screw in limb 2 are similar to those in Table 1. In Table 1, the actuated screw $\$_{11}^{1}$ is replaced by $\$_{21}^{1}$, transmission wrench screw $\$_{T 1}$ is replaced by $\$_{T 2}$. For axis directions of all kinematic pairs, $x$ is replaced by $y, y$ is replaced by $z$, and $z$ is replaced by $x$.

The construction method of limb 2 is the same as that of limb 1, just replace all the subscripts in Table 2 as follows: $x$ is replaced with $y, y$ is replaced with $z, z$ is replaced with $x$, and all configurations of limb 2 can be obtained.

\subsection{Configuration synthesis of limb 3}

Assuming that the output motion along the $z$ direction of the moving platform is only provided by the limb 3 , the reciprocal product of the transmission wrench screw acting on the moving platform and the output twist screw in the $z$ axis direction is not zero, and the reciprocal product of the output twist screw in the other two directions is equal to zero. The derivation method of transmission wrench screw of limb 3 and the construction method of the limb are the same as those of limb 1, just replace the subscript of all the kinematic pairs in Table 2 with the following: $x$ is replaced with $z, y$ is replaced with $x, z$ is replaced with $y$.

\subsection{Construction example of closed-loop units}

According to the closed-loop unit construction method proposed above, this paper select four kinds of basic decoupled limb configuration with compact structure and excellent performance in Table 2. Take the types of driving pair and the screws of kinematic pairs connected to the moving platform into account, a variety of closed-loop units under the same decoupled limb configuration with different kinematic pair layout are obtained, as shown in Table 4.

Furthermore, 10 typical fully decoupled 3T PMs with high stiffness are constructed by connecting the closed-loop units containing the basic decoupled limbs with the moving and fixed platforms, as shown in Table 5.

\section{Synthetic example and verification of a fully decoupling three translational parallel mechan ism with high stiffness}

The basic decoupled limb of the PRPR with a connectivity of 4 is selected as the optimal limb, and the P pair connected to the fixed platform is selected as the driving pair. Three closed-loop units are constructed and connected with the moving and fixed platforms to obtain the fully decoupled 3P2(RPR) PM with high stiffness, as shown in Figure 6. 
Table4 Criterion for construction of closed-loop unit for three translational fully decoupled parallel mechanisms

\begin{tabular}{|c|c|c|c|c|c|c|}
\hline $\begin{array}{l}\text { Type of } \\
\text { driving } \\
\text { pair }\end{array}$ & $\begin{array}{l}\text { Screw type of } \\
\text { kinematic pair } \\
\text { connected to the } \\
\text { moving platform }\end{array}$ & $\begin{array}{c}\text { Basic } \\
\text { decoupled } \\
\text { limb } \\
\text { configuration }\end{array}$ & $\begin{array}{l}\text { The relationship } \\
\text { between the kinematic } \\
\text { pair of the constructed } \\
\text { limb connecting the } \\
\text { moving platform and the } \\
\text { kinematic pair of the } \\
\text { basic decoupled limb } \\
\text { connecting the moving } \\
\text { platform }\end{array}$ & $\begin{array}{l}\text { The relationship } \\
\text { between the driving } \\
\text { pair of the } \\
\text { constructed limb } \\
\text { and the driving pair } \\
\text { of the basic } \\
\text { decoupled limb }\end{array}$ & $\begin{array}{l}\text { Whether } \\
\text { to add } \\
\text { redundant } \\
\text { actuation }\end{array}$ & Instance \\
\hline \multirow{4}{*}{$\begin{array}{l}\text { Driving } \\
\text { pair is a } \\
\text { prismatic } \\
\text { pair }\end{array}$} & $\begin{array}{l}\text { The kinematic pair } \\
\text { connected to the }\end{array}$ & \multirow[b]{2}{*}{ PRRR } & coaxial & share & No & $\begin{array}{l}\text { Table } 5- \\
\text { (1) }\end{array}$ \\
\hline & $\begin{array}{l}\text { moving platform in the } \\
\text { basic decoupled limb is } \\
\text { a revolute pair }\end{array}$ & & parallel & parallel & Yes & $\begin{array}{c}\text { Table 5- } \\
\text { (2) }\end{array}$ \\
\hline & \multirow{2}{*}{$\begin{array}{l}\text { The kinematic pair } \\
\text { connected to the } \\
\text { moving platform in the } \\
\text { basic decoupled limb is } \\
\text { a prismatic pair }\end{array}$} & \multirow[b]{2}{*}{ PRRP } & share & share & No & $\begin{array}{c}\text { Table } 5- \\
\text { (3) }\end{array}$ \\
\hline & & & coaxial & parallel & Yes & $\begin{array}{l}\text { Table } 5- \\
\text { (4) }\end{array}$ \\
\hline \multirow{6}{*}{$\begin{array}{l}\text { Driving } \\
\text { pair is a } \\
\text { revolute } \\
\text { pair }\end{array}$} & \multirow{3}{*}{$\begin{array}{l}\text { The kinematic pair } \\
\text { connected to the } \\
\text { moving platform in the } \\
\text { basic decoupled limb is } \\
\text { a revolute pair }\end{array}$} & \multirow{3}{*}{ RRRRR } & parallel & coaxial & Yes & $\begin{array}{l}\text { Table } 5- \\
\text { (5) }\end{array}$ \\
\hline & & & parallel & parallel & Yes & $\begin{array}{l}\text { Table 5- } \\
\text { (6) }\end{array}$ \\
\hline & & & parallel & share & No & $\begin{array}{c}\text { Table } 5- \\
\text { (7) }\end{array}$ \\
\hline & \multirow{3}{*}{$\begin{array}{l}\text { The kinematic pair } \\
\text { connected to the } \\
\text { moving platform in the } \\
\text { basic decoupled limb is } \\
\text { a prismatic pair }\end{array}$} & \multirow{3}{*}{ RRRRP } & share & parallel & Yes & $\begin{array}{c}\text { Table } 5- \\
\text { (8) }\end{array}$ \\
\hline & & & coaxial & parallel & Yes & $\begin{array}{l}\text { Table } 5- \\
\text { (9) }\end{array}$ \\
\hline & & & parallel & coaxial & Yes & $\begin{array}{c}\text { Table 5- } \\
\text { (10) }\end{array}$ \\
\hline
\end{tabular}

Table5 Typical fully decoupled 3T PMs

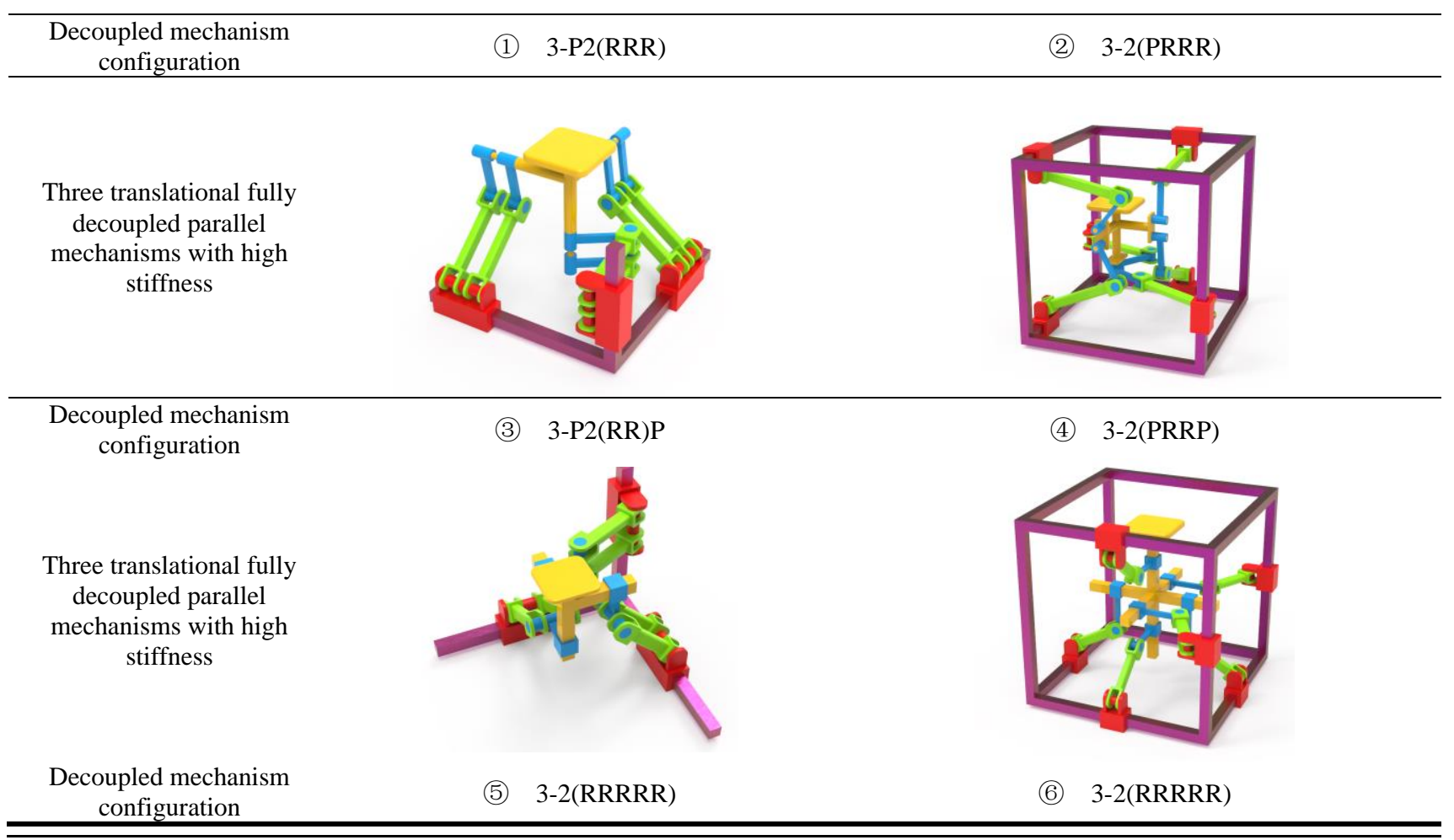


Three translational fully decoupled parallel mechanisms with high stiffness

Three translational fully decoupled parallel mechanisms with high

Decoupled mechanism configuration

$$
\text { stiffness }
$$

Decoupled mechanism configuration

Three translational fully decoupled parallel mechanisms with high stiffness

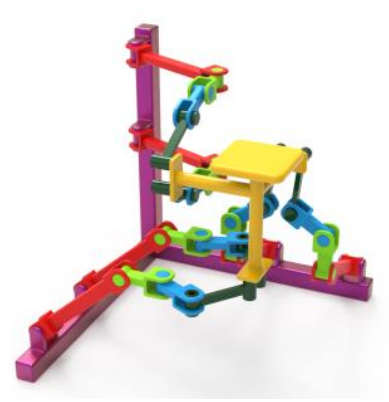

(7) 3-P2(RRRR)

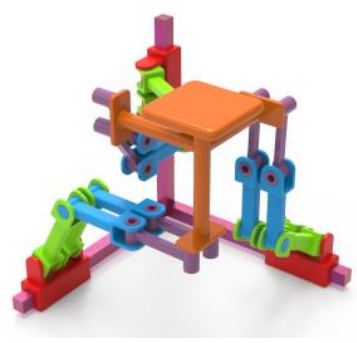

(9) 3-2(RRRRP)

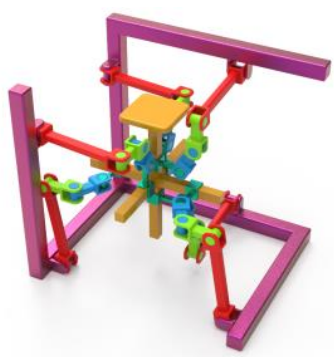

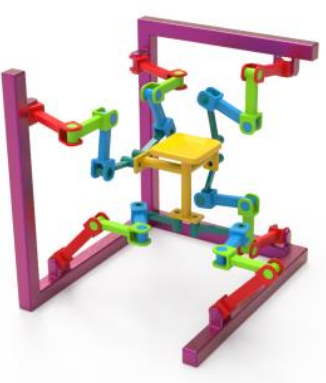

(8) 3-2(RRRR)P

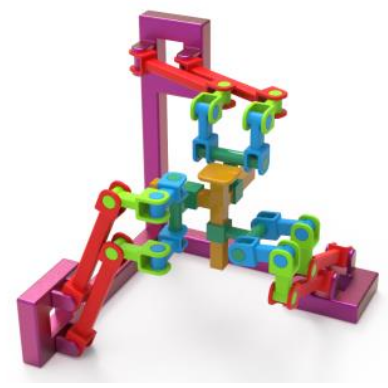

(10) 3-2(RRRRP)

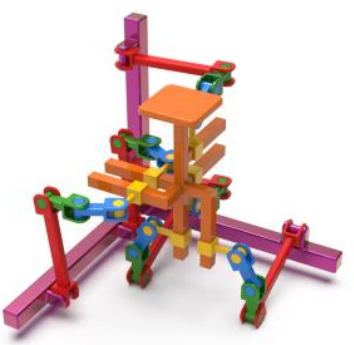

\subsection{Verification of DOF}

The screw system of closed-loop unit 1 is

$$
\begin{aligned}
& \$_{11}=\left(\begin{array}{lllll}
0 & 0 & 0 ; 1 & 0 & 0
\end{array}\right) \\
& \$_{12}=\left(\begin{array}{lllll}
1 & 0 & 0 ; 0 & e_{1} & f_{1}
\end{array}\right) \\
& \$_{13}=\left(\begin{array}{lllll}
0 & 0 & 0 ; 0 & e_{2} & f_{2}
\end{array}\right) \\
& \$_{14}=\left(\begin{array}{lllll}
1 & 0 & 0 ; 0 & e_{3} & f_{3}
\end{array}\right) \\
& \$_{15}=\left(\begin{array}{lllll}
0 & 0 & 0 ; 1 & 0 & 0
\end{array}\right) \\
& \$_{16}=\left(\begin{array}{lllll}
1 & 0 & 0 ; 0 & e_{1} & f_{1}
\end{array}\right) \\
& \$_{17}=\left(\begin{array}{lllll}
0 & 0 & 0 ; 0 & e_{2} & f_{2}
\end{array}\right) \\
& \$_{18}=\left(\begin{array}{lllll}
1 & 0 & 0 ; 0 & e_{3} & f_{3}
\end{array}\right)
\end{aligned}
$$

The reciprocal screw system of closed-loop unit 1 is

$$
\begin{aligned}
& \boldsymbol{\$}_{11}^{\mathrm{r}}=\left(\begin{array}{lllll}
0 & 0 & 0 ; 0 & 1 & 0
\end{array}\right) \\
& \boldsymbol{\$}_{12}^{\mathrm{r}}=\left(\begin{array}{lllll}
0 & 0 & 0 ; 0 & 0 & 1
\end{array}\right)
\end{aligned}
$$

The screw system of closed-loop unit 2 is

$$
\begin{aligned}
& \$_{21}=\left(\begin{array}{lllll}
0 & 0 & 0 ; 0 & 1 & 0
\end{array}\right) \\
& \$_{22}=\left(\begin{array}{lllll}
0 & 1 & 0 ; d_{4} & 0 & f_{4}
\end{array}\right) \\
& \$_{23}=\left(\begin{array}{lllll}
0 & 0 & 0 ; d_{5} & 0 & f_{5}
\end{array}\right) \\
& \$_{24}=\left(\begin{array}{lllll}
0 & 1 & 0 ; d_{6} & 0 & f_{6}
\end{array}\right) \\
& \$_{25}=\left(\begin{array}{lllll}
0 & 0 & 0 ; 0 & 1 & 0
\end{array}\right) \\
& \$_{26}=\left(\begin{array}{lllll}
0 & 1 & 0 ; d_{4} & 0 & f_{4}
\end{array}\right) \\
& \$_{27}=\left(\begin{array}{lllll}
0 & 0 & 0 ; d_{5} & 0 & f_{5}
\end{array}\right) \\
& \$_{28}=\left(\begin{array}{lllll}
0 & 1 & 0 ; d_{6} & 0 & f_{6}
\end{array}\right)
\end{aligned}
$$

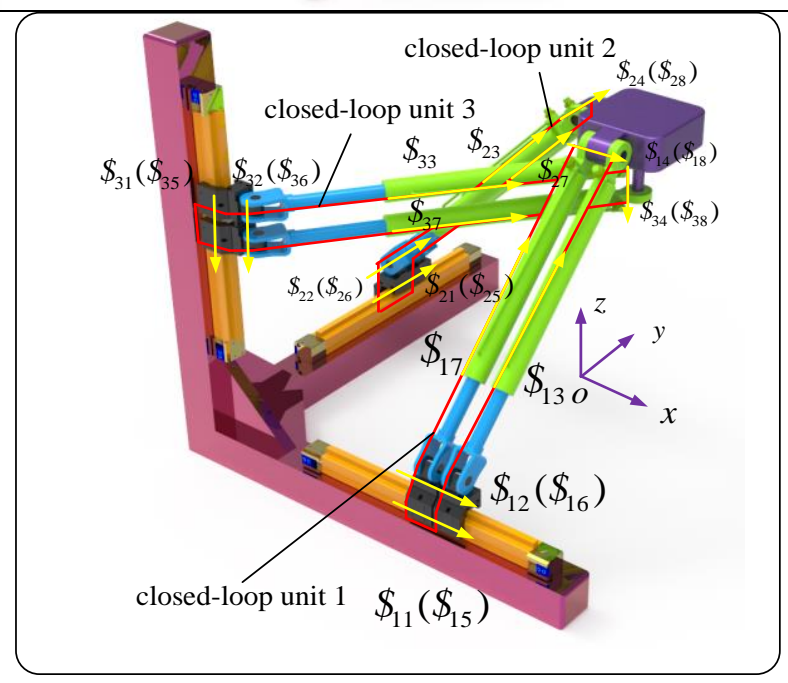

Figure 6 3-P2(RPR) fully decoupled parallel mechanism with closed-loop unit

The reciprocal screw system of closed-loop unit 2 is

$$
\begin{aligned}
& \boldsymbol{\$}_{21}^{\mathrm{r}}=\left(\begin{array}{lllll}
0 & 0 & 0 ; 1 & 0 & 0
\end{array}\right) \\
& \boldsymbol{\$}_{22}^{\mathrm{r}}=\left(\begin{array}{lllll}
0 & 0 & 0 ; 0 & 0 & 1
\end{array}\right)
\end{aligned}
$$

The screw system of closed-loop unit 3 is 


$$
\begin{aligned}
& \$_{31}=\left(\begin{array}{lllll}
0 & 0 & 0 ; 0 & 0 & 1
\end{array}\right) \\
& \$_{32}=\left(\begin{array}{lllll}
0 & 0 & 1 ; d_{7} & e_{7} & 0
\end{array}\right) \\
& \$_{33}=\left(\begin{array}{lllll}
0 & 0 & 0 ; d_{8} & e_{8} & 0
\end{array}\right) \\
& \$_{34}=\left(\begin{array}{lllll}
0 & 0 & 1 ; d_{9} & e_{9} & 0
\end{array}\right) \\
& \$_{35}=\left(\begin{array}{lllll}
0 & 0 & 0 ; 0 & 0 & 1
\end{array}\right) \\
& \$_{36}=\left(\begin{array}{lllll}
0 & 0 & 1 ; d_{7} & e_{7} & 0
\end{array}\right) \\
& \$_{37}=\left(\begin{array}{lllll}
0 & 0 & 0 ; d_{8} & e_{8} & 0
\end{array}\right) \\
& \$_{38}=\left(\begin{array}{lllll}
0 & 0 & 1 ; d_{9} & e_{9} & 0
\end{array}\right)
\end{aligned}
$$

The reciprocal screw system of closed-loop unit 3 is

$$
\begin{aligned}
& \boldsymbol{\$}_{31}^{\mathrm{r}}=\left(\begin{array}{lllll}
0 & 0 & 0 ; 1 & 0 & 0
\end{array}\right) \\
& \boldsymbol{\$}_{32}^{\mathrm{r}}=\left(\begin{array}{lllll}
0 & 0 & 0 ; 0 & 1 & 0
\end{array}\right)
\end{aligned}
$$

The DOF of the 3-P2(RPR) mechanism is calculated by the modified Kutzbach-Grubler formula

$$
M=6(n-g-1)+\sum_{\mathrm{i}=1}^{\mathrm{g}} f_{i}+v=3
$$

where $M$ is the DOF of the mechanism, $n$ is the total number of components containing the racks, $g$ is the number of kinematic pairs, $f_{i}$ is the DOF of the ith kinematic pair, and $v$ is the number of redundant constraints for a multi-loop PM without considering the common constraints.

The above reciprocal screws of the closed-loop units show that the constraint screws of the three closed-loop units restrict the rotational DOF of the moving platform in three directions, thus verifying that $3-\mathrm{P} 2(\mathrm{RPR})$ has three translational DOF.

\subsection{Validation of Jacobian Matrix}

Assuming that the input distance of each driving pair on the base is $l_{1}, l_{2}, l_{3}$, and the corresponding output distance of the moving platform is $x, y, z$ respectively. According to the structural characteristics of the 3-P2(RPR) decoupled PM, the relationship between input and output can be expressed as

$$
\begin{aligned}
& x=l_{1} \\
& y=l_{2} \\
& z=l_{3}
\end{aligned}
$$

The time-derivatives of the above equations are given by

$$
\begin{aligned}
& \dot{x}=\dot{l}_{1} \\
& \dot{y}=\dot{l}_{2} \\
& \dot{z}=\dot{l}_{3}
\end{aligned}
$$

Eq. (34) can be written in the matrix form

$$
\left[\begin{array}{l}
\dot{x} \\
\dot{y} \\
\dot{z}
\end{array}\right]=\left[\begin{array}{lll}
1 & 0 & 0 \\
0 & 1 & 0 \\
0 & 0 & 1
\end{array}\right]\left[\begin{array}{l}
\dot{l}_{1} \\
\dot{l}_{2} \\
\dot{l}_{3}
\end{array}\right]
$$

The Jacobian matrix of the mechanism can be obtained

$$
J=\left[\begin{array}{lll}
1 & 0 & 0 \\
0 & 1 & 0 \\
0 & 0 & 1
\end{array}\right]
$$

The Jacobian matrix is a unit matrix, and the Jacobian determinant is always a fixed value. There is no singular position in the working space of the moving platform, and there will be no movement distortion. One output motion is controlled by only one corresponding input motion, which also verifies that the $3-\mathrm{P} 2(\mathrm{RPR}) \mathrm{PM}$ has three translational full decoupled characteristics, and the mechanism is completely isotropic in the entire working space. Under the condition of satisfying the decoupled characteristics, the isotropic PM has excellent kinematic characteristics and mechanical properties at the same time.

\subsection{Stiffness verification of a three translational fully decoupled parallel mechanism with closed-loop units}

The spatial composite deformation (bending, stretching and torsion deformation) of the connecting rod under the action of the generalized restraining force (including the driving force) is obtained. Firstly, the limb generalized constraint force stiffness matrix is solved. Then, combining with the static balance equation of the moving platform and the principle of virtual work, the overall stiffness matrix of the mechanism is obtained.

The analytical expression of 3-PRPR PM stiffness matrix is

$$
K_{3-\mathrm{PRPR}}=J_{1} K_{1} J_{1}^{\mathrm{T}}+J_{2} K_{2} J_{2}^{\mathrm{T}}+J_{3} K_{3} J_{3}^{\mathrm{T}}
$$

where $J_{i}$ represents the Jacobian matrix of the generalized constraint force of the $i$ th limb, and $K_{i}$ represents the generalized constraint force stiffness matrix of the $i$ th limb.

The analytical expression of the stiffness matrix of 3 P2(RPR) PM is

$$
\begin{array}{r}
K_{3-\mathrm{P} 2(\mathrm{RPR})}=J_{1} K_{1} J_{1}^{\mathrm{T}}+J_{2} K_{2} J_{2}^{\mathrm{T}}+J_{3} K_{3} J_{3}^{\mathrm{T}} \\
+J_{1}^{\prime} K_{1}^{\prime} J_{1}^{\prime \mathrm{T}}+J_{2}^{\prime} K_{2}^{\prime} J_{2}^{\prime \mathrm{T}}+J_{3}^{\prime} K_{3}^{\prime} J_{3}^{\prime \mathrm{T}}
\end{array}
$$

where $J_{i}^{\prime}$ represents the Jacobian matrix of the generalized constraint force of the constructed limb in the $i$ th loop. The direction of the constraint force of constructed limb and the basic decoupled limb are the same, so it can be deduced that $J_{i}=J_{i}^{\prime}$. The length and cross-sectional dimensions of the two RPR limbs in the loop are the same, so $K_{i}=K_{i}^{\prime}$ can be deduced. Therefore,

$$
K_{3-\mathrm{P} 2 \text { (RPR) }}=2 K_{3-\mathrm{PRPR}}
$$

The displacement of each driving pair is taken as $q_{i}=0.05-0.4 \mathrm{~m}$, and the length of each rod of the RPR limb is $l_{i}=0.5-0.75 \mathrm{~m}$. For compare, the same constraint conditions of 3-PRPR and 3-P2(RPR) are given, and the same cross sections are selected in the $Z$-axis direction $(Z=0.45 \mathrm{~m})$ in the working space. The distribution of the maximum eigenvalues of the static stiffness matrix of 3-PRPR PM and 3-P2(RPR) PM is shown in Figure 7.

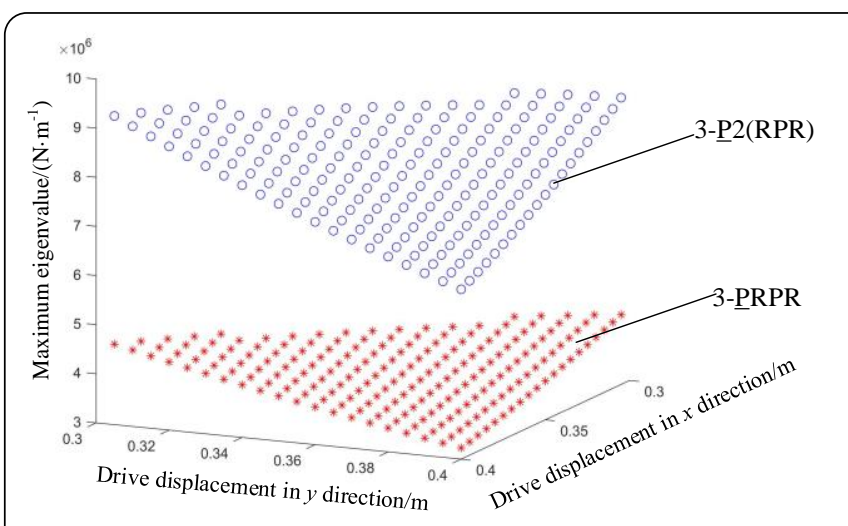

Figure 7 The distribution of the maximum eigenvalues of the static stiffness matrix of 3-PRPR PM and mechanism 3-P2 (RPR) PM

According to Figure 7, maximum eigenvalue of the stiffness matrix of the new 3-르(RPR) PM is double larger than that of 
the 3-PRPR decoupled PM in the workspace. The addition of the closed-loop units doubles the stiffness of the PM, which verifies the 3-P2(RPR) PM has higher stiffness.

When the prismatic pair of 3-PRPR close to the moving platform is used as the driving pair, the mechanism is a coupling mechanism. After the above derivation, it can be seen that the number of constructed limbs (n) in the 3-Pn(RPR) loop is proportional to the stiffness of the mechanism. In order to analyze the stiffness difference between the 3-PRPR coupling mechanism [36] and the 3-Pn(RPR) decoupled mechanism, the $\mathrm{VM}$ index is used as the stiffness performance evaluation index, as shown in eq. (42). That is, the reciprocal of the virtual work done by the external force. This performance index relates the value of the stiffness index to the external force and presents the ability of the mechanism to resist the external force in a specific direction.

$$
V M=\frac{1}{W^{T} \delta \xi}=\frac{1}{W^{T} K^{-1} W}
$$

where $K$ represents the stiffness matrix of the mechanism, $W$ represents the external force on the moving platform. The two mechanisms use the same structural parameters. Given the external load $\mathrm{W}$ is

$$
W=\left[\begin{array}{llllll}
100 N & -100 N & -100 N & 10 N \cdot m & -10 N \cdot m & 10 N \cdot m
\end{array}\right]^{\mathrm{T}}
$$

When $\mathrm{n}=2$, the same cross-sectional parameters of the $\mathrm{Z}$ axis ( $Z=0.45 \mathrm{~m}$ ) are selected, and the VM index comparison diagram of the two mechanisms in the working space is shown in the Figure 8.

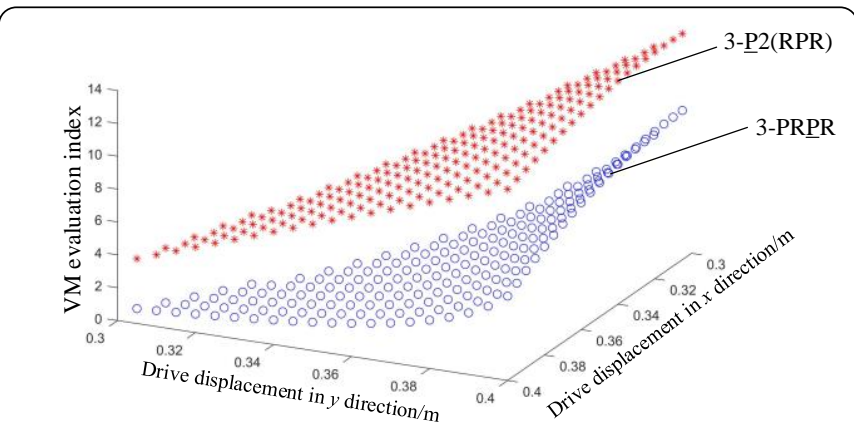

Figure 8 VM index of 3-P2(RPR) PM and 3-PRPR PM

Figure 8 shows that compared with the coupling mechanism 3-PRPR, the VM index of the new decoupled mechanism 3$\underline{P} 2$ (RPR) is significantly improved. The VM value of 3$\underline{\mathrm{P}} 2$ (RPR) is above the value of 3-PRPR at any position, which indicates that 3-P2 $(\mathrm{RPR})$ has less deformation and greater stiffness under the same load. According to the rigidity requirement, the rigidity characteristic of the mechanism can be improved by adjusting the number of $n$, and the precision of the mechanism will be improved as well.

\subsection{Application prospect of decoupled parallel mechanism with high stiffness}

For the sake of the accurate fatigue life prediction of a car, there is often a durability testing before the car leaving the factory. The test usually carried out by a wheel coupled durability test platform. However, the platform can only simulate the excitation in the vertical direction, and ignore the actual excitation in the lateral and tangential directions, resulting in low road spectrum reproduction accuracy. Since the road map is very complex, the application of the highly coupled PM will increase the difficulty of control and cannot meet the requirements of road map reproduction accuracy. Therefore, a decoupled PM can be used to solve this problem[37].

However, the existing fully decoupled PMs have poor rigidity and cannot be applied to high-precision automobile durability testing. The high-stiffness PM with closed-loop units synthesized in this paper, as shown in Figure 6, has the characteristics of decoupled and high stiffness, which can provide lateral and tangential excitation for durability testing, and improve the accuracy of road spectrum reproduction. It can also reduce the difficulty of control, so that it has a better application prospect in automobile durability testing.

\section{Conclusions}

(1) A new synthesis method of a fully decoupled 3T PM with closed-loop units and high rigidity was proposed.

(2) Aiming at the problem of poor stiffness of decoupled PMs, a closed-loop unit construction method was developed, and more than 50 new configurations of high-rigidity fully decoupled 3T PMs with closed-loop units were synthesized.

(3) The kinematics and stiffness analyses verify the correctness of the 3T PM with fully decoupled and high rigidity characteristic. The addition of the closed-loop units makes the rigidity performance of the orthogonal decoupled PM better than that of the orthogonal coupling PM. This paper provided new ideas for the synthesis of the high stiffness and fully decoupled PMs with closed-loop units for automobile durability testing.

\section{Declaration}

\section{Acknowledgment \\ Not applicable}

\section{Funding}

This research was sponsored by the National Natural Science Foundation of China under Grant 51775475, the militarycivilian integration project of Hebei Province under Grant 2020B030.

\section{Availability of data and materials}

The datasets supporting the conclusions of this article are included within the article.

\section{Authors' contributions}

Shihua Li was in charge of the whole research; Sen Wang wrote the manuscript; Haoran Li discussed and read the manuscript, Yongjie Wang assisted with the analysis and validation. Shuang Chen was responsible for the grammar check and error correction of the paper.

\section{Competing interests}

No conflict of interest exits in the submission of this manuscript, and manuscript is approved by all authors for publication 


\section{Consent for publication}

We would like to submit the enclosed manuscript entitled “ Type Synthesis of Fully Decoupled Three Translational Parallel Mechanism with Closed-loop Units and High Stiffness", which we wish to be considered for publication in "Chinese Journal of Mechanical Engineering".

\section{Ethics approval and consent to participate}

I would like to declare on behalf of my co-authors that the work described was original research that has not been published previously, and not under consideration for publication elsewhere, in whole or in part. All the authors listed have approved the manuscript that is enclosed.

\section{Authors' Information}

Shihua Li, born in 1966, is currently a professor, doctoral supervisor at School of Mechanical Engineering, Yanshan University, China. He received his $\mathrm{PhD}$ degree from Yanshan University, China, in 2004. His main research interest is the theory and application of parallel robot. Tel:15703377377; Email: shli@ysu.edu.cn

Sen Wang, born in 1995, is currently a $\mathrm{PhD}$ candidate at School of Mechanical Engineering, Yanshan University, China. He received his master degree on Mechanical engineering in Yanshan University, China, in 2020.

E-mail: 2743824384@qq.com

Haoran Li, born in 1998, is currently a master candidate at School of Mechanical Engineering, Yanshan University, China. E-mail: lhr_764709262@163.com

Yongjie Wang, born in 1997, is currently a master candidate at School of Mechanical Engineering, Yanshan University, China. E-mail: $\underline{1811012262 @ q q . c o m}$

Shuang Chen, born in 1983, is currently a PhD candidate at School of Mechanical Engineering, Yanshan University, China. She is a lecturer at Qing Gong College, North China University of Science and Technology.

E-mail: 157200223@qq.com

\section{References}

[1] B B Lian,T Sun, Y M Song, et al. Stiffness Analysis and Experiment of a Novel 5-DOF Parallel Kinematic Machine Considering Gravitational Effects. International Journal of Machine Tools \& Manufacture, 2015, 95: 8296.

[2] J Ramirez, J Wollnack. Flexible Automated Assembly Systems for Large CFRP-structures Procedia Technology,2014, 15: 447-455.

[3] J P Kobler, J Kotlarski, J Oltjen, et al. Design and Analysis of A Head-mounted Parallel Kinematic Device for Skull Surgery. International Journal of Computer Assisted Radiology and Surgery, 2012, 7(1): 137-149.
[4] L Nurahmi, P Putrayudanto, G Wei, et al. Geometric Constraint-Based Reconfiguration and Self-Motions of a Four-CRU Parallel Mechanism. J. Mechanisms Robotics, 2021, 13(2): 1-21.

[5] Z Huang, Q C Li, H F Ding. Theory of Parallel Mechanisms. Netherlands: Springer, 2013.

[6] Y Li, Y Zhang, L Zhang. A New Method for Type Synthesis of 2R1T and 2T1R 3-DOF Redundant Actuated Parallel Mechanisms with Closed Loop Units. Chinese Journal of Mechanical Engineering, 2020, 33(1). 144-167.

[7] G X Zhang, D H Zheng, J W Guo, et al. Dynamic Modeling and Mobility Analysis of the 3-R(RRR)R+R Antenna Mechanism. Robotica, 2021, 39(8): 1485-1503.

[8] D X Zeng, Y Hou, W J Lu, et al. Comparative Analysis of Characteristics of the Coupled and Decoupled Parallel Mechanisms. Chinese Journal of Mechanical Engineering, 2010, 23(4): 468-476.

[9] H T Liu, K Xu, H P Shen, et al. Type Synthesis of 1T2R P arallel Mechanisms Using Structure Coupling-Reducing Method. Chinese Journal of Mechanical Engineering, 201 9, 32(1): 1-10.

[10] S Briot, I A Bonev. Pantopteron-4 a New 3T1R Decouple d Parallel Manipulator for Pick-and-Place Applications. M echanism \& Machine Theory, 2010, 45(5): 707-721.

[11] Y Zhang, H Liu, W Xin. Kinematics analysis of a novel p arallel manipulator. Mechanism \& Machine Theory, 2009, 44(9): 1648-1657.

[12] Q Jin, T Yang. Synthesis and Analysis of a Group of 3-De gree-of-Freedom Partially Decoupled Parallel Manipulato rs. Journal of Mechanical Design, 2004,126(2): 301-306.

[13] S W Qu, R Q Li, C H Ma, et al. Type synthesis for lowermobility decoupled parallel mechanism with redundant co nstraints. Journal of Mechanical Science and Technology, 2021, 35(6): 2657-2666.

[14] F Zhang, Z B Zhou, J G Yang. Topological Synthesis of F ully Decoupled Parallel Manipulators by Screw Formulati on of Jacobian Matrix. Advanced Materials Research, 201 1, 189-193: 1409-1412.

[15] Q Jin, T L Yang. Synthesis and Analysis of a Group of 3 Degree-of-Freedom Partially Decoupled Parallel Manipul ators. Journal of Mechanical Design,2004,126(2): 301-30 6.

[16] R Lin, W Guo, F Gao. Type Synthesis of a Family of Nov el Four, Five, and Six Degrees-of-Freedom Sea Lion Ball Mechanisms With Three Limbs. Journal of Mechanisms a nd Robotics,2016,8(2): 1-12.

[17] G Gogu. Structural Synthesis of Parallel Robots: Part 2: Translational Topologies with Two and Three Degrees of Freedom. Netherlands: Springer, 2009.

[18] G Yang, I M Chen, W Chen, et al. Kinematic Design of a Six-DOF Parallel-Kinematics Machine with DecoupledMotion Architecture. IEEE Transactions on Robotics, 2004, 20(5):876-887.

[19] V Glazunov. Design of Decoupled Parallel Manipulators by Means of the Theory of Screws. Mechanism \& Machine Theory, 2010, 45(2):239-250.

[20] X W Kong, C M Gosselin, P L Richard. Type Synthesis of Parallel Mechanisms with Multiple Operation Modes. Journal of Mechanical Design, 2006, 129(6): 595-601.

[21] X Liu, J Wang. Some New Parallel Mechanisms Containing the Planar Four-Bar Parallelogram. The International Journal of Robotics Research,2003, 22(9): 717-732.

[22] W M Li, F Gao, J J Zhang. R-CUBE, a Decoupled Parallel Manipulator only with Revolute Joints. Mechanism \& Machine Theory, 2005, 40(4): 467-473.

[23] S Briot, I A Bonev. Pantopteron: A New Fully Decoupled 3DOF Translational Parallel Robot for Pick-and-Place Applications. Journal of Mechanisms and Robotics,2009, 1(2): 795-810.

[24] S Awtar, J Ustick, S Sen. An XYZ Parallel-Kinematic Flexure Mechanism with Geometrically Decoupled 
Degrees of Freedom. Journal of Mechanisms and Robotics, 2012, 5(1): 1-7.

[25] M Carricato, V Parenti-Castelli. Singularity-Free FullyIsotropic Translational Parallel Mechanisms. International Journal of Robotics Research, 2002, 21(2): 161-174.

[26] S Briot, V Arakelian, S Guégan. PAMINSA: a New Family of Decoupled Parallel Manipulators. Mechanism \& Machine Theory, 2009, 44(2): 425-444.

[27] X Jin, Y Fang, H Qu, et al. A Class of Novel 2T2R and 3T2R Parallel Mechanisms with Large Decoupled Output Rotational Angles. Mechanism \& Machine Theory, 2017, 114: $156-169$

[28] X T Sun, W H Chen, R Zhou, et al. A Decoupled 2-DOF Flexure-based Micropositioning Stage with Large Travel Ranges. Robotica, 2014, 32(5): 677-694.

[29] F Gao, Y Zhang, W M Li. Type Synthesis of 3-DOF Reducible Translational Mechanisms. Robotica, 2005, 23(23): 239-245.

[30] Y B Zhang, H Z Liu, X Wu. Kinematics Analysis of a Novel Parallel Manipulator. Mechanism \& Machine Theory, 2009, 44(9): 1648-1657.

[31] S W Qu, R Q Li, S P Bai. Type Synthesis of 2T1R Decoupled Parallel Mechanisms Based on Lie Groups and Screw Theory. Mathematical Problems in Engineering.
2017(1): 1-11.

[32] Q Jin, T L Yang. Theory for Topology Synthesis of Parallel Manipulators and its Application to ThreeDimension-Translation Parallel Manipulators. Journal of Mechanical Design, 2004, 126(4): 625-639.

[33] W M Li, F Gao, J J Zhang. R-CUBE, A Decoupled Parallel Manipulator only with Revolute Joints. Mechanism \& Machine Theory, 2005, 40(4): 467-473.

[34] F Q Zhao, S Guo, H B Qu. Novel High Stiffness Redundant Parallel Mechanism with Closed-loop Units. Journal of Mechanical Engineering, 2017, 53(9): 30-37. (in Chinese)

[35] X J Liu, F G Xie, J S Wang. Fundamental of Parallel Robotic Mechanisms. Beijing: Higher Education Press, 2018. (in Chinese)

[36] Q Cheng, R Q Li. Design and Workspace Optimization of 3-CPR Parallel Mechanism. Journal of Mechanical Transmission, 2017, 41(4): 163-167. (in Chinese)

[37] H B Gong, L Yao. A Review on Technology Development of Indoor Road Simulation Test. Chinese Journal of Automotive Engineering, 2018, 8(1): 72-78. (in Chinese) 